\title{
An Algorithmic Approach to Management of COVID-19 Associated Multisystem Inflammatory Syndrome in Children
}

\author{
Roxana Mansour Ghanaie ${ }^{1}$, Abdollah Karimi (iD ${ }^{1}$, Zahra Pourmoghaddas ${ }^{2}$, Shahnaz Armin ${ }^{1}$, Seyed \\ Alireza Fahimzad ${ }^{1}$, Fatemeh Fallah ${ }^{1}$, Katayoun Tayeri ${ }^{3}$, Mohammad Reza Sabri ${ }^{4}$, Hamid Rahimi ${ }^{5}$, Ali \\ Akbar Zeinaloo ${ }^{6}$, Seyedeh Mahsan Hoseinialfatemi ${ }^{1}$, Vahid Ziaee ${ }^{7}$, Reza Shiari (iD ${ }^{8}$, Bahador \\ Mirrahimi ${ }^{1}$, Ali Saffaei ${ }^{9}$ and Sedigheh Rafiei Tabatabaei ${ }^{1, *}$ \\ ${ }^{1}$ Pediatric Infections Research Center, Research Institute for Children's Health, Shahid Beheshti University of Medical Sciences, Tehran, Iran \\ ${ }^{2}$ Child Growth and Development Research Center, Isfahan University of Medical Sciences, Isfahan, Iran \\ ${ }^{3}$ Iranian Research Center of HIV \& AIDS, Tehran University of Medical Sciences, Tehran, Iran \\ ${ }^{4}$ Pediatric Cardiovascular Research Center, Cardiovascular Research Institute, Isfahan University of Medical Sciences, Isfahan, Iran \\ ${ }^{5}$ Department of Pediatrics, Isfahan University of Medical Sciences, Isfahan, Iran \\ ${ }^{6}$ Department of Pediatric Cardiology, Children's Medical Center (The Pediatric Center of Excellence), Affiliated to Tehran University of Medical Sciences, Tehran, Iran \\ ${ }^{7}$ Division of Pediatric Rheumatology, Pediatrics Center of Excellence, Children's Medical Center, Tehran University of Medical Sciences, Tehran, Iran \\ ${ }^{8}$ Department of Pediatric Rheumatology, Mofid Children's Hospital, Shahid Beheshti University of Medical Sciences, Tehran, Iran \\ ${ }^{9}$ Student Research Committee, Department of Clinical Pharmacy, School of Pharmacy, Shahid Beheshti University of Medical Sciences, Tehran, Iran \\ "Corresponding author: Pediatric Infections Research Center, Research Institute for Children's Health, Shahid Beheshti University of Medical Sciences, Tehran, Iran. Email: \\ srt.tabatabaei@gmail.com
}

Received 2020 October 27; Revised 2020 December 09; Accepted 2020 December 10.

Keywords: Coronavirus, COVID-19, SARS-CoV-2, MIS-C, Multisystem Inflammatory Syndrome in Children

\section{Introduction}

COVID-19 is a viral infection that causes severe acute respiratory syndrome coronavirus-2 (SARS-CoV-2). In adults, severe respiratory symptoms are the most common clinical presentation (1). Although initial studies suggested that children will experience a mild illness during the acute infection (2), recent studies reported the emergence of a new SARS-CoV-2 infection-related phenomenon of a significant hyperinflammatory response in previously healthy asymptomatic children (3). This new phenomenon, termed "multisystem inflammatory syndrome in children (MISC)," has been reported in individuals younger than 19 years. MISC has different clinical presentations, including gastrointestinal problems, mild or absent respiratory symptoms, cardiac disease, rash, and conjunctivitis. Some patients also present features of myocarditis with cardiogenic shock and toxic shock syndrome. It is not clear whether MISC is a consequence of the Covid-19 or not (4). Concerning the viral pathogenicity, SARS-CoV-2 first gains entry into the host cells through its owns proteins. The clinical features of human coronavirus and adenovirus infections can even mimic Kawasaki disease (KD), the etiology of which has not been elucidated yet but has been suggested to be due to an infection (especially of viral origin) based on its acute onset, limited course, and wave-like spread as well as the age of affected patients. Additionally, adenovirus, coronavirus, and parainfluenza virus type 3 may also be isolated from patients with KD as a co-infection. However, it is well-documented that the increased levels of acute-phase reactants, white blood cell, and neutrophil counts, induced by bacterial agents (e.g., superantigens), are involved in KD development (5-7). Some have suggested that the replication of respiratory viruses in the respiratory epithelium and the increase in the IgA-producing plasma cell response could explain the pathogenesis of KD. Interestingly, an increase in the host's IgA response has also been found in the first stage of SARS-COV-2 infection, suggesting a link between these two diseases. The hypothesis that molecular mimicry leads to immunological cross-reactivity, similar to that occurs in rheumatic fever, also worth considering.

These findings suggest a possible link between respiratory viruses, including earlier generations of coronaviruses, and those responsible for $\mathrm{KD}$, and may concern whether SARS-COV-2 infection increases the risk of KD (8). Herein, our multidisciplinary approach to MISC is presented, and current knowledge on the case definition and clinical manifestations are discussed. Finally, a protocol for the diagnosis and treatment of this syndrome is proposed. 


\section{Arguments}

\subsection{Case Definition}

Because MISC is not well defined, the current study aimed to consider a unique definition in this regard. Although according to the literature, this syndrome may strike at any time, it often occurs one to six weeks following the SARS-CoV-2 infection (9). MISC can present with cardiac manifestations, as in Kawasaki disease; however, the involvement of coronary arteries is rare.

MISC can occur with evidence of involvement or even multiple organ failure, including neurological involvement, hyperferritinemia, and cardiogenic or vasogenic shock. Primarily studies mentioned significant gastrointestinal complications, such as diarrhea, vomiting, and severe abdominal pain. MISC can be presented as Kawasaki like disease with complete, incomplete, or atypical manifestation, or can be first diagnosed by nonspecific presentations like shock or gastrointestinal, skin, respiratory, or neurological signs and symptoms.

\subsection{Initial Interventions}

For those who met the abovementioned criteria, early workup should be performed, and for those with Kawasakilike disease, echocardiography should be provided. For all patients, the epidemiological link to SARS-COV-2 infection was evaluated, and preventive and treatment protocols were implemented. After 48 to 72 hours, patients should be evaluated again. It worth noting that those with fever were discharged.

\subsection{Differential Diagnosis}

1- Viral infections (enterovirus, adenovirus, EpsteinBarr virus, measles virus, cytomegalovirus), bacterial infections (Rocky Mountain spotted fever, scarlet fever, bacterial cervical lymphadenitis, leptospirosis, urinary tract infection, meningococcemia), rheumatic diseases (systemic-onset juvenile idiopathic arthritis, Behçet's disease, rheumatic fever), toxic shock syndrome, serum sickness, staphylococcal scalded skin syndrome, macrophage activation syndrome, drug hypersensitivity reactions, Stevens-Johnson syndrome, and aseptic meningitis (10).

2- KD in patients with fever for more than 5 days who met at least four of the following classical findings: conjunctivitis, changes in the limbs, exanthema, erythema of the oral mucosa and lips, and cervical lymphadenopathy as well as complete KD in those with 2 or 3 of the above clinical findings with at least 3 of these laboratory pieces of evidence, that is, white blood cell (WBC) count $\geq 15,000 / \mu \mathrm{L}$, normochromic anemia due to age-mediated changes, normocytic, platelet count $\geq 450,000 / \mu \mathrm{L}$ after seven days of illness, non-neutrophilic (sterile) pyuria due to urethritis in $\mathrm{KD}$, serum alanine aminotransferase $>50$ units/L, Serum albumin $\leq 3 \mathrm{~g} / \mathrm{dLin}$ addition to elevated acute-phase reactants (C-reactive protein (CRP) $\geq 3 \mathrm{mg} / \mathrm{dL}$ or erythrocyte sedimentation rate (ESR) $\geq 40 \mathrm{~mm} /$ hour) (11).

3- For children with gastrointestinal symptoms, information regarding their travel history to infected areas, presence in crowded places, and history of contact with a definite or suspected case of COVID-19 patient should be obtained; however, during pandemics, a clear history of contact may not be found.

4- Lymphocytopenia for different age groups: absolute lymphocyte count $<3000 / \mathrm{mL}$ in infants (1 - 12 months), < $2000 / \mathrm{mL}$ in children $1-5$ years of age, and $<1100 / \mathrm{mL}$ in children $>5$ years of age).

5- Crimean-Congo hemorrhagic fever (CCHF): As Iran is an endemic region for CCHF (12), it should be considered in every child with headache, high fever, back pain, vomiting, red eyes, diarrhea, and bleeding. Besides, petechiae (red spots) and history of contact with livestock or CCHF patients should be evaluated.

\subsection{Further Evaluations}

All of the above patients with persistent fever in addition to one of the following signs and symptoms should be further evaluated: 1- shock or hypotension; 2heart failure or myocarditis; 3- acute gastroenteritis; 4unexplained ascites; 5- hepatitis with or without jaundice; 6- splenomegaly; 7- skin rash (popular vesicular petechiae/purpura); 8- coagulopathies; 9- encephalitis (loss of consciousness, seizure, cranial involvements) PLUS at least one of the following findings: 1-cytopenia; or 2- pancytopenia (lymphopenia according to age, thrombocytopenia, neutropenia, or neutrophilia). In such patients, toxic shock syndrome, bacterial septic shock, and CCHF should be evaluated, in addition to $\mathrm{KD}$, according to the clinical signs and symptoms.

\subsection{Criteria for Multisystem Inflammatory Syndrome in Chil- dren}

Concerning MIS-C evaluation, all of the following criteria should be met.

1- An individual of $<19$ years of age; 2 - All of the following clinical and laboratory features: (i) Fever of 38 degrees or more for at least 24 hours; (ii) Multisystem $(\geq 2)$ organ involvement: cardiovascular (revealed by shock, increased troponin, increased B-type natriuretic peptide, abnormal echocardiography, arrhythmia), renal (acute kidney injury, renal failure), respiratory(pneumonia, adult respiratory 
distress syndrome, pulmonary embolism), hematologicallike coagulopathy, gastrointestinal(diarrhea, ileus, gastrointestinal bleeding, increase liver enzyme), dermatological (erythroderma or other rashes, mucositis), or neurological (seizure, aseptic meningitis, stroke, decreased level of consciousness); (iii) Evidence of a clinically severe illness that requires hospitalization; (iii) Evidence of inflammation as revealed by at least one of the following criteria: increased CRP, D-dimer, ESR, ferritin, interleukin-6, procalcitonin, lactic acid dehydrogenase, fibrinogen, neutrophils, or decreased absolute lymphocyte or albumin count; 3- No alternative justifying diagnoses; 4- Positive RTPCR, serology or antigen tests for current and recent infections or a history of exposure to an individual with suspected (e.g., recommended to isolate) or confirmation of COVID-19 diagnosis 4 weeks before the onset of symptoms. During pandemics, exposure can be considered even in those without clear contact history.

\subsection{Specific Evaluations Suspected to Have MISC}

For all patients, the following additional clinical and paraclinical evaluations should be performed: 1- Frequent monitoring of all abovementioned signs and symptoms and excluding the focal infection; 2- Stabilizing the hemodynamic status; 3- Obtaining the echocardiography, arterial blood gas measurements, electrocardiography, and sonography (if gastrointestinal signs and symptoms present); 4- Obtaining the cultures from sterile body fluids (e.g., blood, cerebrospinal fluid, ascites) or from wounds and the throat; 5-Conducting liver enzyme and function tests as well as checking the levels of electrolytes, $\mathrm{Ca}, \mathrm{Mg}$, acute-phase reactants, triglycerides, ferritin, fibrinogen, albumin, and troponin; and 6- conducting nasopharyngeal/oropharyngeal RT-PCRs for SARS-CoV-2.

All of the abovementioned laboratory tests should be repeated according to the patient's condition. Scoring for CCHF based on the national guidelines should be kept in mind.

\subsection{Pharmacological Interventions}

The severity assessment should be performed and accordingly, patients should be divided into mild, moderate, and severe groups. In the case of any suspicion of infection, appropriate empirical antibiotics should be initiated.

Mild: those with minimal organ injury (13), no or minimal respiratory support, and no vasoactive requirement. Those with mild or isolated organ damage, coronary involvement, progressive or significant oxygen requirement, and need for vasoactive therapy should be categorized as moderate. In severe cases, one may have moderate to severe organ damage, including ventricular dysfunction, non-invasive or invasive ventilatory support, and need for vasoactive therapy.

Intravenous Immunoglobulin (IVIG) and aspirin are recommended to all patients with Kawasaki-like disease. Additionally, IVIG and methylprednisolone should be considered in moderate and severe cases or those with a progressive pattern. It is noted that deep vein thrombosis should be prevented by administering low-molecularweight heparin (Enoxaparin) to all PICU-admitted (pediatric intensive care unit) patients of $>12$ years old. (It should be considered in the moderate and severe groups, too)

\subsection{Refractory Patients}

In cases with no appropriate responses to the abovementioned interventions, intravenous immunoglobulin can be re-administered. Additionally, biological agents, such as infliximab, anakinra, and tocilizumab, may be considered on a patient-case basis (14). The proposed protocol is shown in Figure 1.

\subsection{Recommendations}

1-In the time of the COVID-19 pandemic, each child with two organ failures should be evaluated for MISC; 2 - In the time of the COVID-19 pandemic, each child with Kawasakilike disease should be evaluated for MISC; 3-If there is a suspicion of MISC along with hemodynamic instability or cardiac failure, the intensity of the disease will be more severe; hence, emergency interventions are necessary.

\section{Conclusion}

There are reports indicating that COVID-19 associated MISC can worsen rapidly. Therefore, the early implementation of a higher level of care (i.e., transferring to the PICU) is necessary. As discussed herein, a multidisciplinary approach with an expert group for those convention the disease classification is of crucial importance for achieving successful outcomes.

\subsection{Discharge Criteria and Follow-Up}

Children who are well and have a stable cardiac function and do not have pyrexia for 24 hours can be discharged from the hospital. Children with MISC should be followed up in 24 - 72 hours after discharge and have a further followup with echocardiography6 weeks after discharge. They should be visited in follow-up by a multidisciplinary team.

Strongly encourage a multidisciplinary team and protocol to uniformly screen, diagnose and treat MIS-C catered to institutional resources and expertise. Any suspected 
Non-specific: presenting with shock or at least one of these symptoms like that do not meet the criteria for Kawasaki disease: gastrointestinal include abdominal pain, respiratory, or neurological symptoms

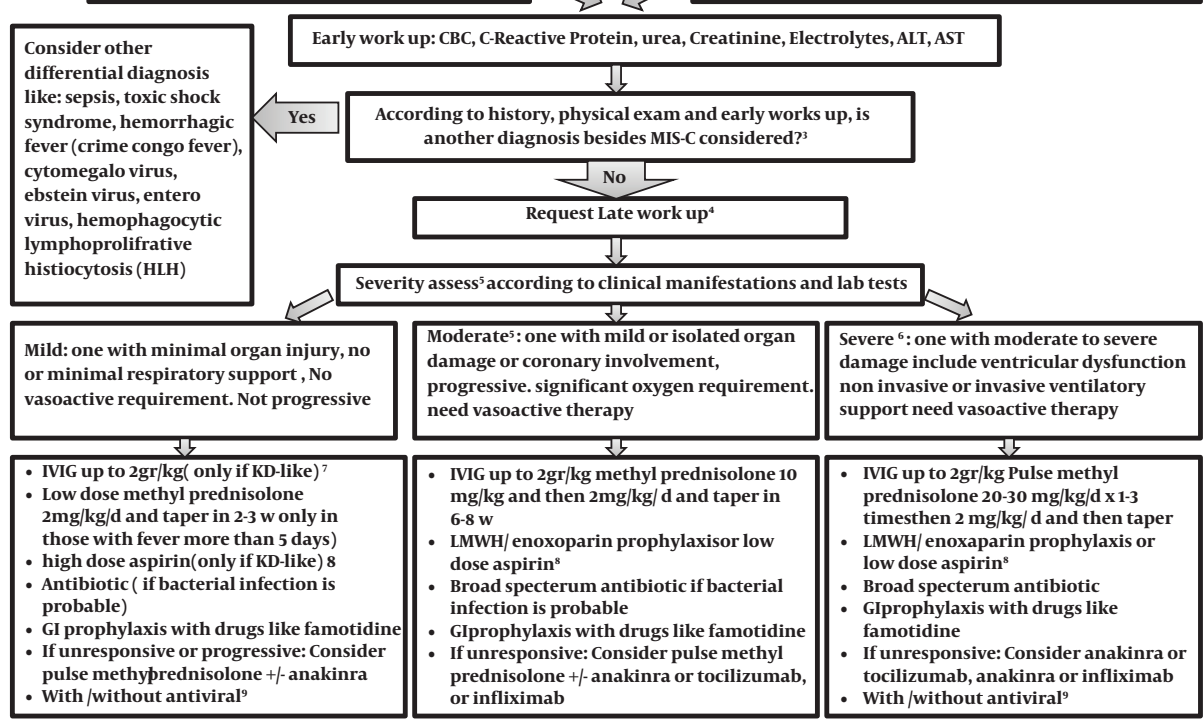

Figure 1. Management of children with suspected COVID 19-associated multisystem inflammatory syndrome in children (MISC). ${ }^{1}$ classifiedaccording to American Heart Association criteria (15); ${ }^{2}$ If $>1$ of these signs and symptoms: 1- petechial, purpuric, vesicular, or bullous Rash, 2-predominant GI symptoms: Diarrhea, abdominal pain, vomiting, 3- Neurologic Symptoms: decreased level of consciousness, seizure, cranial nerve involvement, 4- Hemodynamic instability, heart failure, or carditis, 5- Splenomegaly, 6- increase liver enzyme, hepatitis with or without jaundice, ascites, 7-Coagulopathy, 8-AND $\geq 1$ of the following findings: Bicytopenia or pancytopenia (lymphopenia according to age, thrombocytopenia; 3MIS-C criteria in children; ${ }^{4}$ Second-line investigations: blood gas, lactate, fibrinogen, ferritin, troponin, N-terminal pro-B-type natriuretic peptide, D-dimer, lactate dehydrogenase, SARS-CoV-2 RT-PCR test, and serology, Septic and viral screen (lumbar puncture only if specifically indicated),12-lead electrocardiogram, Chest radiograph, Echocardiogram, Contrast-enhanced CT of the coronary vessels inadvisable for MISC. For children with abdominal pain who have symptoms of MISC, an abdominal ultrasound scan should be the first test to rule out other alternative diagnoses such as appendicitis; ${ }^{5}$ If evidence of coronary artery abnormality; use of IVIG with or without intravenous methylprednisolone $(10-30 \mathrm{mg} / \mathrm{kg})$. If progressive or unresponsive, a natural therapy should be used in children who do not answer to IVIG and methylprednisolone, infliximab is preferred for those with Kawasaki-like presentation and anakinra/ tocilizumab for others. In those small groups with the presentation of HLH, special treatment according to the consultation is recommended; ${ }^{6}$ severe disease can be considered if any of the following physiologic or biochemical features is presented, especially when there are in combination: delayed capillary tube refill time; continual hypotension; persistent tachycardia; requirement for $40 \mathrm{~mL} / \mathrm{kg}$ fluid bolus; oxygen saturation < 92\%, increase in CRP to $300 \mathrm{mg} / \mathrm{L}$ (some suggests $>150 \mathrm{mg} / \mathrm{L}$ ); high troponin, NT-proBNP, ferritin, D-dimer, lactate dehydrogenase, creatinine, more or less fibrinogen, Cardiac symptoms like an atypical electrocardiogram, left ventricular failure or coronary artery aneurysms on echocardiogram (16); ${ }^{7}$ IN KD-like: First-line therapy for all children is IVIG at a dose of $2 \mathrm{~g} / \mathrm{kg}$, the second dose of IVIG is used for children who have not responded to the first dose of the drug. Some experts recommend that high-risk children (those that probably do not respond to IVIG according to kabashi score) or those Less than a year, and those with coronary artery changes; intravenous methylprednisolone (10 - $30 \mathrm{mg} / \mathrm{kg}$; along with IVIG) must be prescribed at first. The second line of treatment is intravenous methylprednisolone (10 - 30 mg/kg) as the subsequent treatment choice for children who remain unwell and febrile $48-72 \mathrm{~h}$ after infusion of intravenous immunoglobulin. Biological therapy is considered as a third-line choice in children who do not reply to IVIG and methylprednisolone. In those with Kawasaki-like phenotype, infliximab is preferred (3). ${ }^{8}$ In cases of Kawasaki-like disease prescribe high dose aspirin (50-80 mg/kg/d) use aspirin till fever persists, and then continue with a low dose for 6 weeks. Use LMWH/enoxaparin as prophylaxis is recommended in moderate and severe cases of MISC and those children older than 12 years old (If hemorrhage and thrombocytopenia are controlled). All children over the age of 12 must wear compression stockings (13). MISC patients with coronary heart involvement or documented thrombosis should receive LMWH/ enoxaparin according to AHA protocol (15); ${ }^{9}$ In children with positive RT-PCR or with presentation consistent with typical covid-19might some experts recommend antiviral like favipiravir/ remdesivir.

PIMS-TS child should be discussed and reviewed by a multidisciplinary team within 24 hours.

Multidisciplinary follow-up at discharge is essential to both understand and monitor disease progression.

References

\section{Acknowledgments}

The authors are thankful to Dr. Vadood Javadi Parvaneh, Dr. Raheleh Assari, Dr. Ehsan Aghaei, Dr. Hamid
Eshaghi, and Dr. Seyed Reza Raeeskaramifor for their cooperation.

\section{Footnotes}

Authors' Contribution: Study concept and design: Roxana Mansour Ghanaie, Abdollah Karimi and Sedigheh Rafiei Tabatabaei, Reza Shiari; Analysis and interpretation of data: Mohammad Reza Sabri, Hamid Rahimi; Drafting of the manuscript: Roxana Mansour Ghanaie, Seyedeh Mahsan Hoseini-Alfatemi; Critical revision of the manuscript 
for important intellectual content: Shahnaz Armin, Seyed Alireza Fahimzad, Fatemeh Fallah, Katayoun Tayeri; Statistical analysis: Bahador Mirrahimi, Ali Saffaei.

Conflict of Interests: The author declare no conflict of interest

Funding/Support: This study was supported by the Research Institute for children's health, Shahid Beheshti University of medical sciences, Tehran, Iran.

\section{References}

1. Lai CC, Shih TP, Ko WC, Tang HJ, Hsueh PR. Severe acute respiratory syndrome coronavirus 2 (SARS-CoV-2) and coronavirus disease-2019 (COVID-19): The epidemic and the challenges. Int J Antimicrob Agents. 2020;55(3):105924. doi: 10.1016/j.ijantimicag.2020.105924. [PubMed: 32081636]. [PubMed Central: PMC7127800].

2. Lu X, Zhang L, Du H, Zhang J, Li YY, Qu J, et al. SARS-CoV-2 infection in children. N Engl J Med. 2020;382(17):1663-5. doi: 10.1056/NEJMc2005073. [PubMed: 32187458]. [PubMed Central: PMC7121177].

3. Hennon TR, Penque MD, Abdul-Aziz R, Alibrahim OS, McGreevy MB, Prout AJ, et al. COVID-19 associated Multisystem Inflammatory Syndrome in Children (MIS-C) guidelines; a Western New York approach. Prog Pediatr Cardiol. 2020:101232. doi: 10.1016/j.ppedcard.2020.101232. [PubMed: 32837142]. [PubMed Central: PMC7244417].

4. Rowley AH. Multisystem inflammatory syndrome in children and kawasaki disease: Two different illnesses with overlapping clinical features. J Pediatr. 2020;224:129-32. doi: 10.1016/j.jpeds.2020.06.057.

5. Giray T, Bicer S, Kucuk O, Col D, Yalvac Z, Gurol Y, et al. Four cases with Kawasaki disease and viral infection: aetiology or association. Infez Med. 2016;24(4):340-4. [PubMed: 28011972].

6. Sundel RP, Newburger JW. Kawasaki disease. Curr Opin Infect Dis. 1992;5(5):664-9. doi: 10.1097/00001432-199210000-00007.

7. Rowley AH, Gonzalez-Crussi F, Shulman ST. Kawasaki syndrome. Rev Infect Dis. 1988;10(1):1-15. doi: 10.1093/clinids/10.1.1. [PubMed: 3281216].
8. Raba AA, Abobaker A. COVID-19 and Kawasaki Disease: An Etiology or Coincidental Infection? Pediatr Infect Dis J. 2020;39(8). e213. doi: 10.1097/INF.0000000000002779. [PubMed: 32467454].

9. Kaushik S, Aydin SI, Derespina KR, Bansal PB, Kowalsky S, Trachtman R. Multisystem inflammatory syndrome in children (MISC) associated with SARS-CoV-2 infection: A multi-institutional study from New York City. J pediatr. 2020. doi: 10.1016/j.jpeds.2020.06.045. [PubMed: 32553861]. [PubMed Central: PMC7293760].

10. Pilania RK, Bhattarai D, Singh S. Controversies in diagnosis and management of Kawasaki disease. World J Clin Pediatr. 2018;7(1):27-35. doi: 10.5409/wjcp.v7.i1.27. [PubMed: 29456929]. [PubMed Central: PMC5803562].

11. Dietz SM, van Stijn D, Burgner D, Levin M, Kuipers IM, Hutten BA et al. Dissecting Kawasaki disease: a state-of-the-art review. Eur J Pediatr. 2017;176(8):995-1009. doi: 10.1007/s00431-017-2937-5. [PubMed: 28656474]. [PubMed Central: PMC5511310].

12. Chinikar S, Ghiasi SM, Moradi M, Goya MM, Shirzadi MR, Zeinali M, et al. Geographical distribution and surveillance of Crimean-Congo hemorrhagic fever in Iran. Vector Borne Zoonotic Dis. 2010;10(7):705-8. doi: 10.1089/vbz.2009.0247. [PubMed: 20854025].

13. Belay E OM, Cheung E, Tremoulet A. Clinical management of multisystem inflammatory syndrome in children (MISC) associated with Coronavirus disease 2019 (COVID-19). Centers for Disease Control and Prevention; 2020. Available from: https://emergency.cdc.gov/coca/calls/ 2020/callinfo_071620.asp.

14. Dastan F, Nadji SA, Saffaei A, Tabarsi P. Tocilizumab administration in a refractory case of COVID-19. Int J Antimicrob Agents. 2020;56(2):106043. doi: 10.1016/j.ijantimicag.2020.106043. [PubMed: 32502524]. [PubMed Central: PMC7264941].

15. McCrindle BW, Rowley AH, Newburger JW, Burns JC, Bolger AF, Gewitz $M$, et al. Diagnosis, treatment, and long-term management of Kawasaki disease: A scientific statement for health professionals From the American heart association. Circulation. 2017;135(17):e92799. doi: 10.1161/CIR.0000000000000484. [PubMed: 28356445].

16. Harwood R, Allin B, Jones CE, Whittaker E, Ramnarayan P, Ramanan A, et al. A national consensus management pathway for paediatric inflammatory multisystem syndrome temporally associated with COVID-19 (PIMS-TS): results of a national Delphi process. Lancet Child Adolesc Health. 2020. doi: 10.1101/2020.07.17.20156075. 